\title{
Generation of HLA-Universal iPSC-Derived Megakaryocytes and Platelets for Survival Under Refractoriness Conditions
}

\author{
Ann-Kathrin Börger,${ }^{1,5^{*}}$ Dorothee Eicke, ${ }^{1,5^{*}}$ Christina Wolf, ${ }^{1}$ Christiane Gras, ${ }^{1,5}$ Susanne Aufderbeck, ${ }^{1}$ \\ Kai Schulze, ${ }^{2}$ Lena Engels, ${ }^{3,5}$ Britta Eiz-Vesper, ${ }^{1}$ Axel Schambach, ${ }^{4,5}$ Carlos A Guzman, ${ }^{2,5}$ Nico Lachmann, ${ }^{4,5}$ \\ Thomas Moritz, ${ }^{4,5}$ Ulrich Martin, ${ }^{3,5}$ Rainer Blasczyk, ${ }^{1,5}$ and Constança Figueiredo $0^{1,5}$
}

${ }^{1}$ Institute for Transfusion Medicine, Hannover Medical School, Hannover, Germany; ${ }^{2}$ Department of Vaccinology and Applied Microbiology, Helmholtz Centre for Infection Research, Braunschweig, Germany; ${ }^{3}$ Leibniz Research Laboratories for Biotechnology and Artificial Organs, Hannover, Germany; ${ }^{4}$ Institute of Experimental Hematology; and ${ }^{5}$ REBIRTH Cluster of Excellence, Hannover Medical School, Hannover, Germany

\begin{abstract}
Platelet (PLT) transfusion is indispensable to maintain homeostasis in thrombocytopenic patients. However, PLT transfusion refractoriness is a common life-threatening condition observed in multitransfused patients. The most frequent immune cause for PLT transfusion refractoriness is the presence of alloantibodies specific for human leukocyte antigen (HLA) class I epitopes. Here, we have silenced the expression of HLA class I to generate a stable HLA-universal induced pluripotent stem cell (iPSC) line that can be used as a renewable cell source for the generation of low immunogenic cell products. The expression of HLA class I was silenced by up to $82 \%$ and remained stable during iPSC cultivation. In this study, we have focused on the generation of megakaryocytes (MK) and PLTs from a HLA-universal iPSC source under feeder- and xeno-free conditions. On d 19, differentiation rates of MKs and PLTs with means of 58\% and $76 \%$ were observed, respectively. HLA-universal iPSC-derived MKs showed polyploidy with DNA contents higher than $4 \mathrm{n}$ and formed proPLTs. Importantly, differentiated MKs remained silenced for HLA class I expression. HLA-universal MKs produced functional PLTs. Notably, iPSC-derived HLA-universal MKs were capable to escape antibody-mediated complement- and cellular-dependent cytotoxicity. Furthermore, HLA-universal MKs were able to produce PLTs after in vivo transfusion in a mouse model indicating that they might be used as an alternative to PLT transfusion. Thus, in vitro produced low immunogenic MKs and PLTs may become an alternative to PLT donation in PLT-based therapies and an important component in the management of severe alloimmunized patients.
\end{abstract}

Online address: http://www.molmed.org

doi: $10.2119 /$ molmed.2015.00235

\section{INTRODUCTION}

Platelet (PLT) refractoriness represents a relevant clinical concern in transfusion medicine because it is associated with adverse effects including an increased risk of bleeding and reduced survival $(1,2)$. In addition, PLT transfusion refractoriness is associated with significant higher health care costs due to prolonged or repetitive hospital stays (3).
The main immune causes leading to PLT transfusion refractoriness are the alloimmunization to HLA or human platelet antigens due to previous transfusion, pregnancy or transplantation. Nevertheless, HLA alloimmunization is accepted to be the major cause of PLT refractoriness. Primarily, immunoglobulin $\mathrm{G}$ antibodies targeting HLA-A and -B are responsible for the development of

${ }^{*} A-K B$ and DE contributed equally to this work.

Address correspondence to Constança Figueiredo, Institute for Transfusion Medicine, Hannover Medical School, Carl-Neuberg-Straße 1, D-30625 Hannover, Germany. Phone: +49511532 971 1; Fax: +49 511532 9701; E-mail: Figueiredo.Constanca@mh-hannover.de. Submitted November 6, 2015; Accepted for publication May 6, 2016; Published Online (www.molmed.org) May 16, 2016.

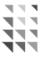

Feinstein Institute

for Medical Research Northwell Health"

refractoriness conditions after PLT transfusion (4-7). Currently, the management of patients suffering of PLT transfusion refractoriness is based on the provision of HLA-matched or cross-matched PLTs. In contrast to the remarkable decline in red blood cell transfusions, the demand on PLTs has significantly increased (8). However, PLT donor shortage, limited shelf life and risk of viral or bacterial infection remains a major obstacle to this practice and have driven the search for alternative sources of blood products. In adults, megakaryocytes (MKs) are localized in the bone marrow and extend long protrusions known as proPLTs, through the sinusoidal blood vessels into the circulation. The feasibility to reproduce thrombopoiesis in vitro from pluripotent stem cells has been demonstrated $(9,10)$. 
Induced pluripotent stem cells (iPSCs) gained plenty of attention in the field of cell therapies including blood pharming, as they may constitute a reliable cell source for the scalable production of several cell types (11). Generally, the use of autologous iPSC-derived grafts has been intended, but the high technical and economic associated efforts constitute a significant hurdle to their clinical application (12). Nevertheless, the use of allogeneic iPSCs is associated with major histocompatibility concerns due to the high variability of HLA and minor histocompatibility antigens (mHAs), which would contribute to the rejection of the potential iPSC-derived cell grafts. Despite some attempts for the establishment of iPSC haplobanks to cover the most frequent HLA haplotypes, it is known from the field of transplantation that even HLA-matched grafts can be rejected due to the presentation of polymorphic mHAs $(13,14)$. Recently, the concept of generating HLA-universal PLTs has been developed using different tools such as RNA interference (RNAi) or Clustered Regularly Interspaced Short Palindromic Repeats / Cas9 (CRISPR/Cas9) $(9,10)$. Previously, we have demonstrated that silencing HLA expression using RNAi prevents an allogeneic immune response in vitro and in vivo. The reduction of HLA expression was shown to be sufficient to inhibit an allogeneic T-cell response. Importantly, the residual expression of HLA class I demonstrated to be crucial to prevent natural killer (NK) cytotoxicity $(10,15,16)$. Thus, in this study, we have generated a HLA-universal iPSC line for the differentiation of low immunogenic products and assessed MK and PLT immunogenicity in vitro and in vivo. Furthermore, we have used it as a cell source for the production of HLA-universal PLTs upon differentiation under defined and xeno-free culture conditions. iPSC-derived HLA-universal PLTs showed the potential to survive under refractoriness conditions in vitro and after transfusion in a mouse model for PLT refractoriness.

\section{MATERIALS AND METHODS}

\section{Generation and Culture of HLA-Universal iPSCs}

The human iPSC line hCBiPSC2 was derived from human cord blood endothelial cells, as previously described (17). iPSCs were adapted to feeder- and xeno-free culture by transfer onto human recombinant Laminin-521-coated plates (BioLamina) and using StemMACS medium (Miltenyi Biotech). Passaging was conducted by detaching the cells with TrypLE Express (Life Technologies), followed by reseeding at a cell density of 50,000 cells $/ \mathrm{cm}^{2}$. For the generation of HLA-universal iPSCs, the cells were transduced with the previously described lentiviral vector pLVTHm encoding a short-hairpin RNA (shRNA) for $\beta 2$-microglobulin silencing (shRNA targeting $\beta 2 \mathrm{~m}$ transcripts or sh $\beta 2 \mathrm{~m}$ ) and the enhanced green fluorescent protein as reporter gene (10). As control, a vector encoding a nonspecific shRNA (shNS) was used as previously described (18). Furthermore, nontransduced iPSCs were used as control. For lentiviral vector transduction, iPSCs were grown to $70 \%$ confluency and transduced in the presence of $8 \mu \mathrm{g} / \mathrm{mL}$ protamine sulfate (Sigma-Aldrich). After $8 \mathrm{~h}$, the viruscontaining medium was removed and fresh medium was added to the cells. Transduction efficiency was calculated by assessing the percentage of GFPexpressing cells. The expression of HLA class I antigen was measured after staining with an anti-HLA class I (w6/32) antibody conjugated with allophycocyanin (APC; AbDSerotec). The expression of the pluripotency markers TRA-1-60 and SSEA-4 was detected by flow cytometry after staining with specific antibodies purchased from BD Biosciences.

\section{Generation of MKs and PLTs From HLA-Universal iPSCs}

For differentiation, nontransduced as well as shNS- and sh $\beta 2$ m-expressing
iPSCs were seeded on Laminin521-coated plates in StemMACS medium. On $\mathrm{d} 0$, the medium was replaced by StemMACS and APEL medium (STEMCELL Technologies; 1:1, vol:vol) containing vascular endothelial growth factor (VEGF; $50 \mathrm{ng} / \mathrm{mL}$ ) and bone morphogenetic protein 4 (BMP4; $50 \mathrm{ng} / \mathrm{mL})$. After $48 \mathrm{~h}$, medium was exchanged to APEL containing VEGF (50 ng/mL) and BMP4 (50 ng/mL). After d 4, the culture medium was replaced with APEL medium containing thrombopoietin (TPO; $50 \mathrm{ng} / \mathrm{mL}$ ), stem cell factor (SCF; $50 \mathrm{ng} / \mathrm{mL}$ ) and interleukin-3 (IL-3; $25 \mathrm{ng} / \mathrm{mL}$ ).

After d 12, APEL medium containing TPO (50 ng/mL) and SCF (50 ng/mL) was used. All growth factors and cytokines were purchased from PeproTech. Cells present in the culture supernatants were harvested gently by rinsing the monolayer at d 12, 19, 26 and 33 and analyzed for the presence of MKs and PLTs, as described below. Separation of the two fractions either containing cells in the size of MKs (large cells) or containing cells in the size of PLTs (small cells + cell fragments) was done with a centrifugal separation step. Harvested cells were first centrifuged at $120 \mathrm{~g}$ for $10 \mathrm{~min}$ to pelletize MKs. PLTs remained in the supernatant were collected and centrifuged again at $740 \mathrm{~g}$ for $10 \mathrm{~min}$. Afterward analysis of cell fractions was done separately.

\section{Characterization of HLA-Expressing and HLA-Universal MKs and PLTs}

Flow cytometry. As previously described (10), iPSC-derived MKs and PLTs were detected via flow cytometry after staining with APC-cyanine 7 (Cy7)-conjugated anti-CD41 (GPIIb), APC-conjugated anti-CD61 (GPIIIa; all from BioLegend) and phycoerythrin (PE)-labeled anti-CD42a (GPIX; BD Biosciences) antibodies. Forward scatter (FSC) and side scatter (SSC) profiles of blood-derived PLTs from healthy volunteers were used to adjust the PLT gate to distinguish PLTs from MKs. In addition, we performed propidium iodide (PI; Sigma-Aldrich) staining for polyploidy 
analysis of MKs. Therefore, cells were stained with APC-Cy7-labeled anti-CD41, washed with phosphate-buffered saline (PBS) and treated with Cytofix/Cytoperm (BD Biosciences). After a second washing step, cells were stained with PI staining solution (10 $\mu \mathrm{g} / \mathrm{mL}$ PI in PBS) containing RNAse A (10 U/mL; Sigma-Aldrich) and acquired by fluorescence-activated cell sorting (FACS). Furthermore, HLA class I expression of nontransduced, shNS- and sh $\beta 2$ m-expressing MKs was evaluated by flow cytometric analysis after staining with an anti-HLA class I (w6/32) antibody conjugated with APC or PE (AbDSerotec).

Real-time polymerase chain reaction. mRNA levels of iPSC-derived progenitor cells and MKs were analyzed as previously described (19). Briefly, total RNA was isolated from cells harvested from differentiation cultures (RNeasy Mini Kit, Qiagen) and reverse transcribed to cDNA using the High-Capacity cDNA Reverse Transcription Kit (Applied Biosystems). Real-time polymerase chain reaction (PCR) was used to measure $\beta 2 \mathrm{~m}$ transcript levels. The GAPDH gene was used as reference standard for normalization of mRNA levels. All real time PCR analyses were performed in triplicates.

Fluorescence microscopy. Morphology of MKs and proPLTs in the differentiation cultures at different stages was assessed with an Olympus IX81 microscope (Olympus). Images were acquired with a digital B/W camera (Olympus) and analyzed with Xcellence Pro image software (Olympus). To visualize polyploid nuclei, MKs were stained with anti-CD61-FITC for $15 \mathrm{~min}$ and afterward stained with 4',6-diamidino-2-phenylindole nucleic acid stain (Invitrogen). Cells were acquired by fluorescence microscopy with adequate filter sets.

PLT activation assays. To assess PLT functionality, iPSC-derived PLTs were separated from MKs as described above and stimulated with adenosine 5 '-diphosphate (ADP, $1 \mathrm{mmol} / \mathrm{L}$ ) and thrombin $(1 \mathrm{U} / \mathrm{mL})$ for $1 \mathrm{~min}$ at room temperature (RT) and immediately stained with anti-CD41-APC-Cy7 and anti-CD62P-PE for $15 \mathrm{~min}$. PLT activation was then analyzed by flow cytometry and compared with the levels of nonstimulated PLTs.

Complement-dependent cytotoxicity assay. hCBiPSC2 were typed positive for HLA-A*02 and HLA-A*29. Lymphocytotoxicity tests were performed with viable nontransduced or shNS- or $\operatorname{sh} \beta 2 \mathrm{~m}$ expressing MKs. Cells were incubated with complement-binding donorspecific anti-HLA-A*02 and anti-HLA-A*29 antibodies (OneLambda). Nonspecific anti-HLA-A*23/24 antibody (OneLambda) was used as negative control. After $1 \mathrm{~h}$ of incubation at RT, $30 \mu \mathrm{L}$ of rabbit complement (Bio-Rad) was added, and after further incubation of 1 h, $25 \mu \mathrm{L}$ of FluoroQuench dye (OneLambda) was added to stain and fix the cells for $20 \mathrm{~min}$. The frequency of lysed cells was analyzed using flow cytometry.

Antibody-dependent cellular cytotoxicity assay. An Antibody-Dependent Cellular Cytotoxicity (ADCC) Reporter Bioassay Kit [ADCC Reporter Bioassay, Complete (WIL2-S); Promega] was used following the manufacturer's instructions. The test was done in a 96-well plate with $12.5 \times 10^{3}$ cells per well expressing either shNS or $\operatorname{sh} \beta 2 \mathrm{~m}$ or nontransduced (target cells). The specific anti-HLA-A*02 antibody (OneLambda) or the antiHLA-ABC antibody (w6/32, Serotec) were applied $(1 \mu \mathrm{g} / \mathrm{mL})$, and the nonspecific antibody targeting HLA-A*23/24 (OneLambda) served as a negative control. WIL2-S cells (effector cells) were added to each well and incubated for $6 \mathrm{~h}$. Then, BioGlo Luciferase Assay Reagent was added to the wells and luminescence was quantified.

PLT transfusion refractoriness mouse model and biodistribution assays. To show the feasibility of in vitro iPSCderived MKs to release PLTs in vivo, a mouse model was used. Three million MKs were transfused by intravenous injection into 8- to 10-wk-old NOD/ SCID/IL-2R $\beta \mathrm{C}^{-/-}$mice previously treated or not with $30 \mu \mathrm{L}$ of an anti-HLA-A*02 antibody (One Lambda) and allowed to distribute within the mice's circulation. Peripheral blood (PB) was drawn before and 1 and $4 \mathrm{~h}$ after injection and analyzed for the presence of human PLTs by flow cytometric analysis. Human PLTs were identified upon staining with antihuman CD42a-PE and CD61-APC, respectively. For biodistribution assays, mice that received human cells were killed 1 or $14 \mathrm{~d}$ after MK transfusion to collect heart, lung, spleen and bone marrow (BM) harvested from the femurs $(n=3)$. All mice were maintained under specific pathogen-free conditions in the animal facility of the Helmholtz Centre for Infection Research. All animal experiments in this study have been performed in agreement with the local government of Lower Saxony (Germany, Az: 33.42505-084/06).

\section{Statistical Analysis}

Statistical analyses were performed using two-tailed $t$ tests run on GraphPad Prism 5 software (GraphPad Software). Levels of significance were expressed as $p$ values $\left({ }^{*} p \leq 0.05,{ }^{* *} p \leq 0.01\right.$ and $\left.{ }^{* * *} p \leq 0.001\right)$.

\section{RESULTS}

\section{Generation of a HLA-Universal iPSC Line}

The high variability of HLA represents a significant hurdle to the application of cell-based products derived from allogeneic iPSCs. In this study, cell cultures showing transduction efficiencies of at least $80 \%$ were used for MK and PLT differentiation. The iPSC transduction using lentiviral vectors encoding for the sequence for $\operatorname{sh} \beta 2 \mathrm{~m}$ resulted in a decrease of HLA class I expression by up to $87 \%$ at transcript level in comparison with the iPSCs expressing shNS (Figure 1A). Furthermore, the downregulation of $\beta 2 \mathrm{~m}$ transcript levels caused a significant reduction of HLA class I protein expression on the cells surface by up to $82 \%$, remaining stable for more than 10 passages (Figure 1B). Of note, HLA class I silenced iPSCs showed comparable levels of the pluripotency markers SSEA-4 and TRA1-60 when compared with nontransduced iPSCs or iPSCs expressing a shNS (Supplementary Figure S1). These data indicate the feasibility to stably silence the expression of HLA class I expression on iPSCs. 
A

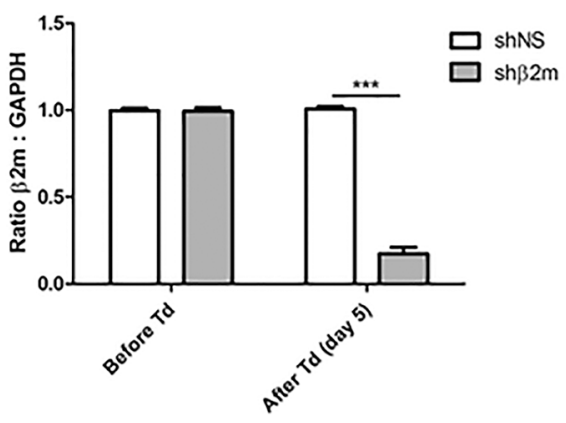

B

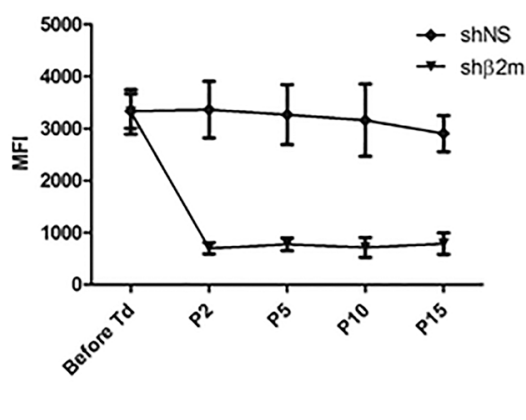

Figure 1. Generation of a HLA-universal iPSC line. iPSCs cultured in monolayer on Laminin-521-coated plates were transduced with a lentiviral vector (pLVTHm) encoding the sequences for a nonspecific shRNA (shNS) or a shRNA targeting $\beta 2 m$ transcripts $(\operatorname{sh} \beta 2 \mathrm{~m})$. (A) Levels of $\beta 2 \mathrm{~m}$ transcripts measured by real time PCR before and $5 \mathrm{~d}$ after cell transduction (Td). GAPDH was used as housekeeping gene to normalize cDNA levels. (B) Expression levels of HLA class I measured by flow cytometric analysis prior transduction and at passages (P) 2, 5, 10 and 15. Graphs show mean \pm standard deviation (SD) $(n=4$, ${ }^{* * *} p \leq 0.001$ )

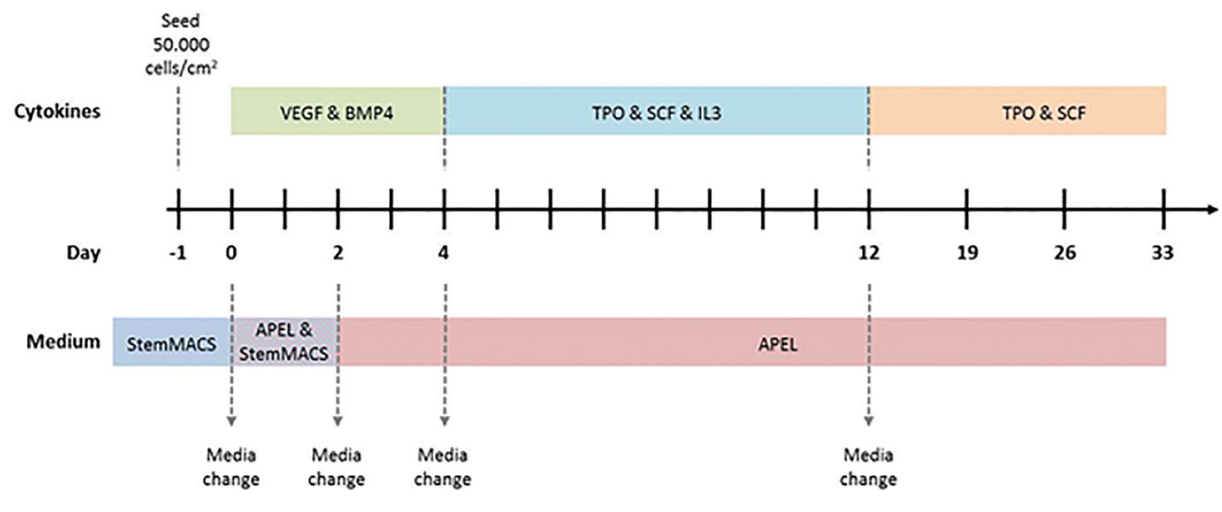

Figure 2. Schematic representation of the iPSC differentiation protocol toward megakaryocytes (MKs) and platelets (PLTs). iPSCs maintained in StemMACS medium were seeded at a density of 50,000 cells $/ \mathrm{cm}^{2}$ on Laminin-52 1-coated plates $1 \mathrm{~d}$ before starting the differentiation. From d 0 to 2, cells were cultured in $1 / 2$ StemMACS medium and $1 / 2$ APEL medium containing vascular endothelial growth factor (VEGF, $50 \mathrm{ng} / \mathrm{mL}$ ) and bone morphogenetic protein 4 (BMP4, $50 \mathrm{ng} / \mathrm{mL}$ ). On d 2, the medium was changed to APEL medium containing VEGF and BMP4 $(50 \mathrm{ng} / \mathrm{mL})$. Cytokines were changed from $\mathrm{d} 4 \mathrm{on}$ to thrombopoietin (TPO, $50 \mathrm{ng} / \mathrm{mL}$ ), stem cell factor (SCF, $50 \mathrm{ng} / \mathrm{mL}$ ) and interleukin-3 (IL-3, $25 \mathrm{ng} / \mathrm{mL}$ ). On d 12, the medium was changed to APEL medium containing TPO and SCF (50 ng/mL). Half of the medium was replaced twice a week. Dotted arrows indicate complete media changes due to modification in cytokine composition.

\section{Differentiation of MKs From HLA-Universal iPSCs Under Defined and Xeno-Free Conditions}

In this study, we focused on the generation of HLA-universal PLTs differentiated from iPSCs. A protocol was established for the differentiation of MKs and PLTs from HLA-universal iPSCs under xeno-free and defined conditions (Figure 2) to facilitate their future translation into clinical application. On d 19, a mean of $58.0 \% \pm 10.9 \%$ of $\mathrm{CD} 41^{+} \mathrm{CD} 42 \mathrm{a}^{+}$
MKs derived from HLA-silenced iPSCs using pLVTHm-encoding $\operatorname{sh} \beta 2 \mathrm{~m}$ were detected in the differentiation cultures (Figures 3A, B). Similar differentiation rates of $51.3 \% \pm 10.5 \%$ or $44.4 \% \pm 14.7 \%$ were detected when MKs were differentiated from shNS-expressing or nontransduced iPSCs, respectively. Furthermore, microscopic analysis showed an increase in ploidy, which was further confirmed by flow cytometric analysis (Figures 3C, D). DNA contents higher than $8 \mathrm{n}$ were detectable in MKs-derived from nontransduced, shNS- or sh $\beta 2 \mathrm{~m}$-expressing iPSCs. Moreover, HLA-universal iPSC-derived MKs showed the capacity to form proPLTs (Figure 3D). In addition, MKs differentiated from iPSCs expressing $\mathrm{sh} \beta 2 \mathrm{~m}$ showed a significant decrease on $\beta 2 \mathrm{~m}$ levels by up to $97.0 \%$ in comparison to shNS-expressing MKs and up to $82.3 \%$ when compared with nonmanipulated MKs (Figure 4A). Furthermore, a mean of reduction of HLA class I expression of $65 \%$ was measured on $\operatorname{sh} \beta 2 \mathrm{~m}$-expressing MKs at d 19 in comparison to nonmanipulated or shNS-expressing MKs (Figure 4B). These data indicate the feasibility to generate MKs from HLA-universal iPSCs with the RNAi-mediated knockdown of HLA class I antigens remaining effective during differentiation.

\section{Generation of HLA-Universal PLTs}

Previously, we have reported the feasibility to generate functional HLA-universal PLTs from CD $34^{+}$progenitor cells isolated from granulocytecolony stimulating factor mobilized healthy donors (19). In this study, we show the feasibility to produce in vitro PLTs derived from HLA-silenced iPSC-derived MKs. To identify and characterize the PLT population, the typical PLT markers CD41 (GPIIb), CD42a (GPIX) and CD61 (GPIIIa) were used (Supplementary Figure S2). The CD41 ${ }^{+}$ population was selected and analyzed for the percentage of $\mathrm{CD} 42 \mathrm{a}^{+} \mathrm{CD} 61^{+}$ co-expression. At d 26, frequencies of $80.7 \% \pm 11.5 \%$ of $\mathrm{CD} 42 \mathrm{a}^{+} \mathrm{CD} 61^{+}$PLTs 
differentiated from $\mathrm{sh} \beta 2 \mathrm{~m}$-expressing MKs were detectable on the supernatants of the differentiated cultures. PLT frequencies of $77.0 \% \pm 11.0 \%$ and $69.6 \%$ \pm 20.6 were measured in the differentiation cultures of MKs derived from shNS-expressing iPSCs or nontransduced iPSCs, respectively (Figure 5). These data indicate that shRNA expression or silencing HLA expression does not impair the differentiation rates of PLTs in vitro.

\section{HLA-Universal PLTs Are Functional}

To evaluate the capacity of HLAuniversal PLTs to respond to extracellular stimuli, we have stimulated them with the classical PLT agonists ADP and thrombin (20). In vitro generated HLA-universal PLTs derived from sh $\beta 2$ m-expressing iPSCs significantly upregulate the expression of CD62P $(43.5 \% \pm 7.8 \% ; p \leq 0.01)$ after activation in comparison to the frequency of CD62P expression detected prior exposure to ADP and thrombin (15.3\% $\pm 0.35 \%)$. Comparable CD62P frequencies were detected when nonmanipulated and shNS-expressing PLTs were stimulated $(30.5 \% \pm 4.6$ and $30.5 \% \pm 5.6 \%$; Figure 6$)$. Furthermore, HLA-universal PLTs were able to form aggregates after stimulation similarly to shNS-expressing or nonmanipulated PLTs (Supplementary Figure S3). These data show that PLTs generated from HLA-universal cell sources are functional.

\section{HLA-Universal MKs Are Able to Escape Antibody-Mediated Complement-Dependent Cytotoxicity}

Severe HLA-alloimmunized patients often develop PLT transfusion refractoriness due to a large panel of reactive antibodies (21). Previously, we have shown that HLA-silenced MKs derived from $\mathrm{CD}_{34}{ }^{+}$progenitor cells are able to survive under refractoriness conditions (10). Here, we have evaluated the capacity of MKs derived from HLA-silenced iPSCs to escape antibody-mediated complement-dependent cytotoxicity (CDC) and ADCC. The iPSCs used for
A

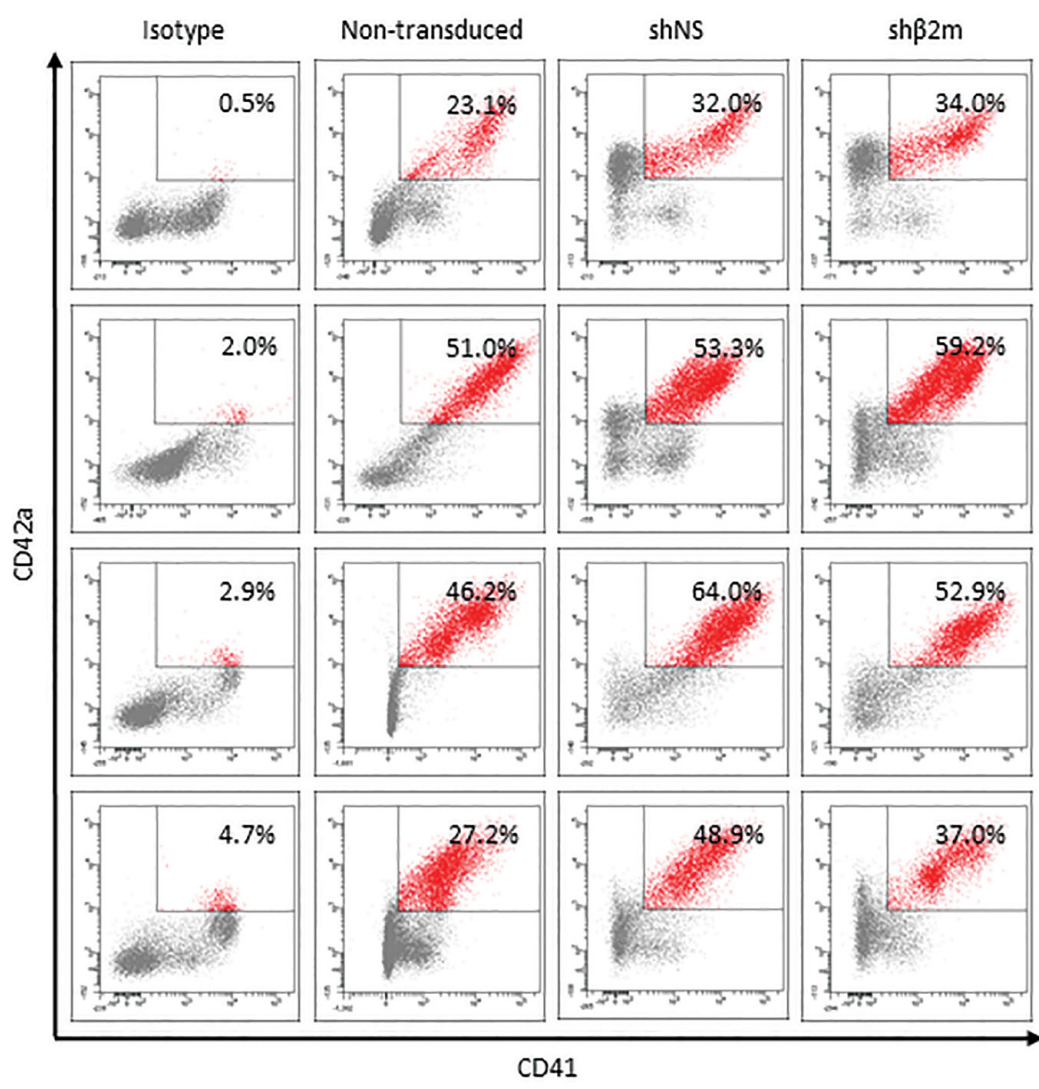

d12

d19

d26

B

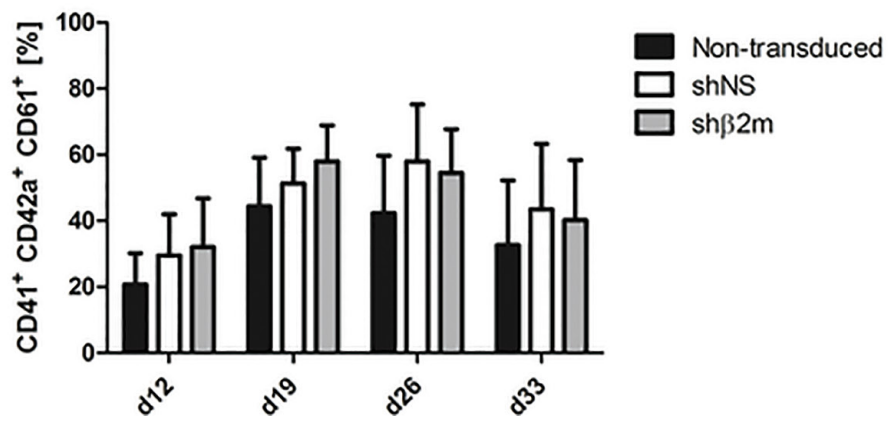

Figure 3. Characterization of iPSC-derived HLA class I-silenced megakaryocytes (MKs). Nontransduced iPSCs and iPSCs expressing either a control nonspecific shNS or a shRNA targeting $\beta 2 m$ transcripts (sh $\beta 2 m$ ) were cultured for $33 \mathrm{~d}$ and analyzed weekly from d 12 for MK differentiation (four time points). iPSCs were transduced with shRNA encoded in a pLVTHm backbone. (A) MKs were identified based on the expression of CD41 (GPIllb) and CD42a (GPIX). Representative flow cytometry dot plots are shown for each condition and time point. (B) Mean and $\mathrm{SD}$ of $\mathrm{CD} 4 \mathrm{l}^{+} \mathrm{CD} 42 \mathrm{a}^{+} \mathrm{CD} 6 \mathrm{I}^{+}$cell frequencies were detected by at least four independent experiments. (C) Polyploidy analysis of shNS or sh $\beta 2 \mathrm{~m}$-expressing $\mathrm{CD}_{4} \mathrm{I}^{+}$cells on d 26 and d 33. Representative flow cytometry histograms are shown. (D) Representative fluorescence microscopy images of MKs exhibiting polyploid nuclei (lower row) and morphological analyses of proPLT-forming MKs (upper row). Representative pictures of nontransduced, shNS or sh $\beta 2 \mathrm{~m}$-expressing MKs on d 20 of differentiation are shown (bright field).

Continued on the next page 


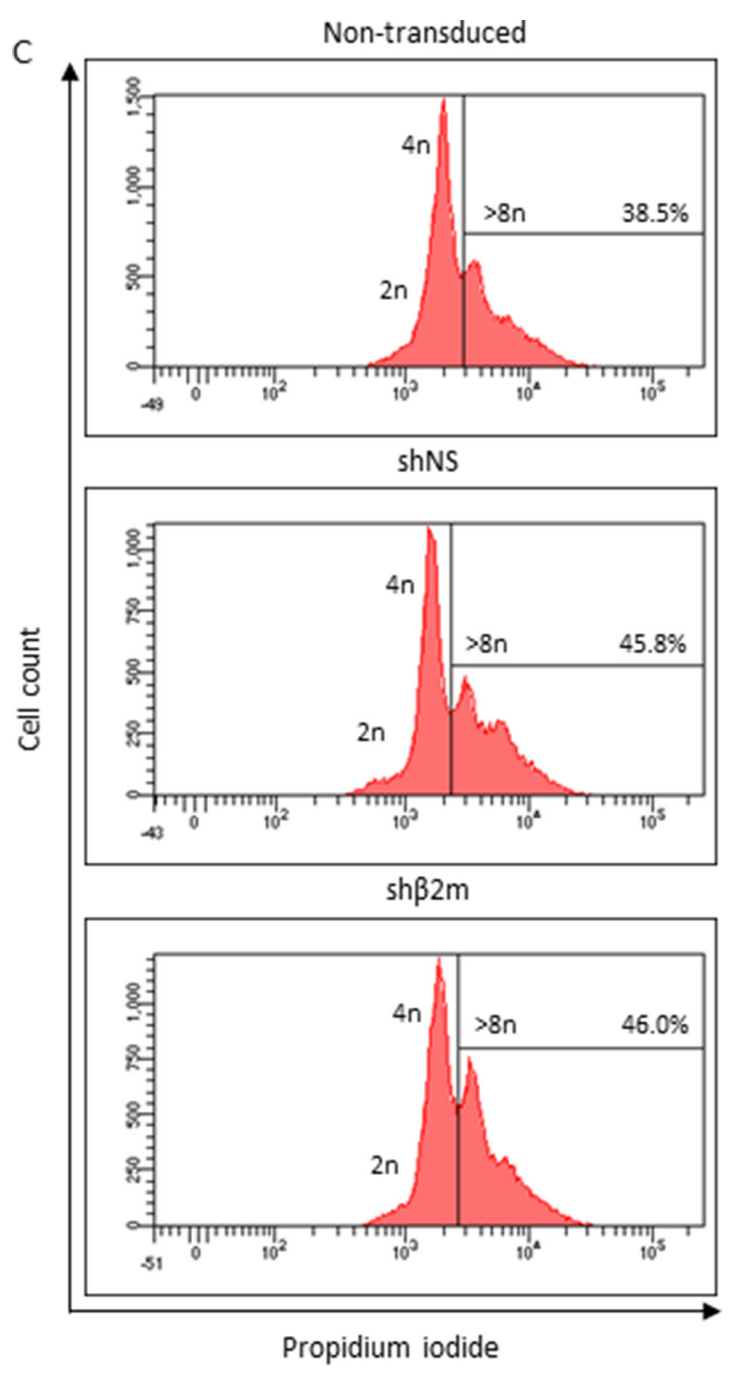

D

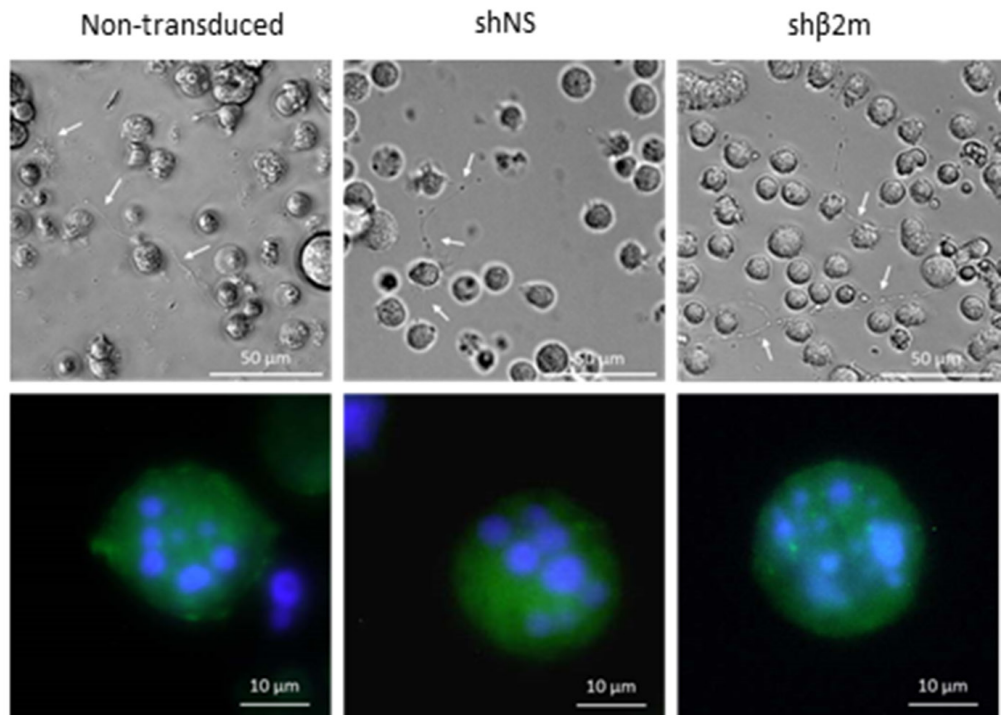

Figure 3. Continued.
MK differentiation were genotyped for HLA-A*02 and HLA-A*29. Therefore, iPSC-derived MKs were incubated with anti-HLA-specific antibodies targeting these antigens. An antibody specific for HLA-A*23/24 was used as control. In comparison to the mean of cell lysis rates of $4.2 \%$ observed when the nonspecific antibody was used (anti-HLA-A*23/24), the incubation of HLA-expressing nonmanipulated MKs resulted in an increase of cell lysis by up to $13.3 \%$ in presence of anti-HLA-A*02 and up to $16.2 \%$ in presence of anti-HLA-A ${ }^{*} 29$. Similarly, shNS-expressing cell lysis rates up to $17.4 \%$ or $13.4 \%$ were detectable after exposure to anti-HLA-A*02 or anti-HLA-A*29, respectively. Conversely, in presence of specific anti-HLA antibodies, cell lysis rates of HLA-universal MKs were comparable to those observed in presence of a nonspecific antibody (Figure 7A). These results indicate that HLA-universal MKs are less targeted by antibody-mediated CDC.

\section{HLA-Universal MKs Are Able to Escape ADCC}

In ADCC assays, MK cell lysis rates were determined as relative luminescence units (RLU). In comparison to the cell lysis rates detectable in presence of a nonspecific anti-HLA-A*23/24, MK lysis rates derived from nontransduced or shNS-expressing iPSCs exposed to specific anti-HLA-A*02 or anti-HLA-ABC antibodies were significantly increased $(p<0.001)$. In contrast, no significant changes in cell lysis rates were observed among $\beta 2 \mathrm{~m}$-silenced MKs incubated with the nonspecific antibody and the specific anti-HLA-A*02 or anti-HLA-ABC antibodies. Remarkably, HLA-silenced MKs showed significantly lower RLU in comparison to fully HLA-expressing $\mathrm{MKs}$ in presence of anti-HLA-A*02 (nontransduced $p<0.05$; shNS $p<0.01$ ) or anti-HLA-ABC antibodies (nontransduced $p<0.001$; shNS $p<0.01$; Figure 7B). These data demonstrate that HLAuniversal MKs also circumvent cellular cytotoxicity induced by anti-HLAspecific antibodies. 
A

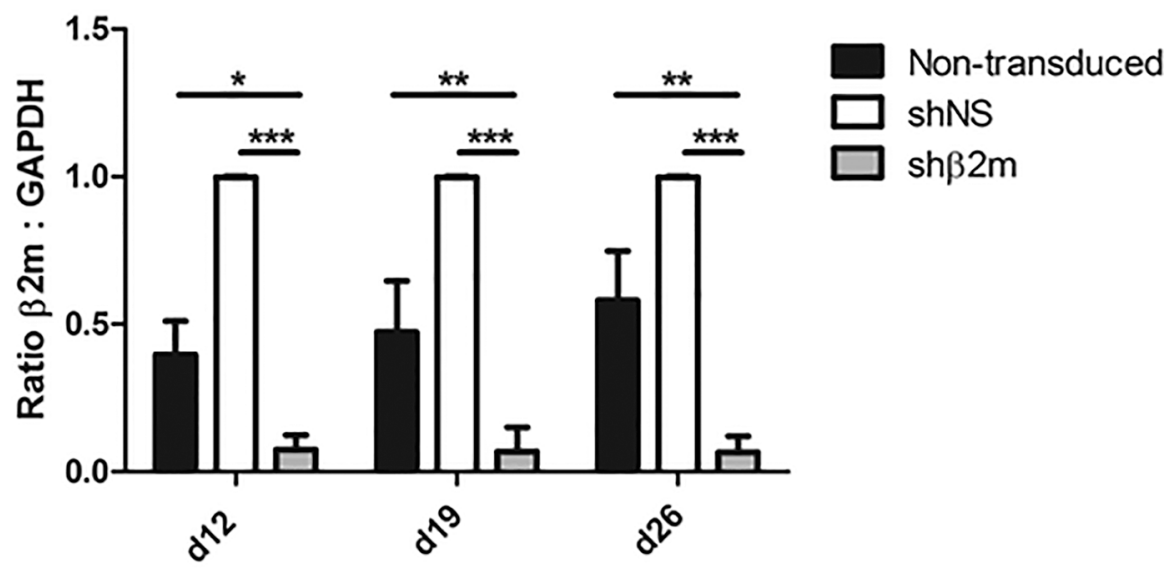

B

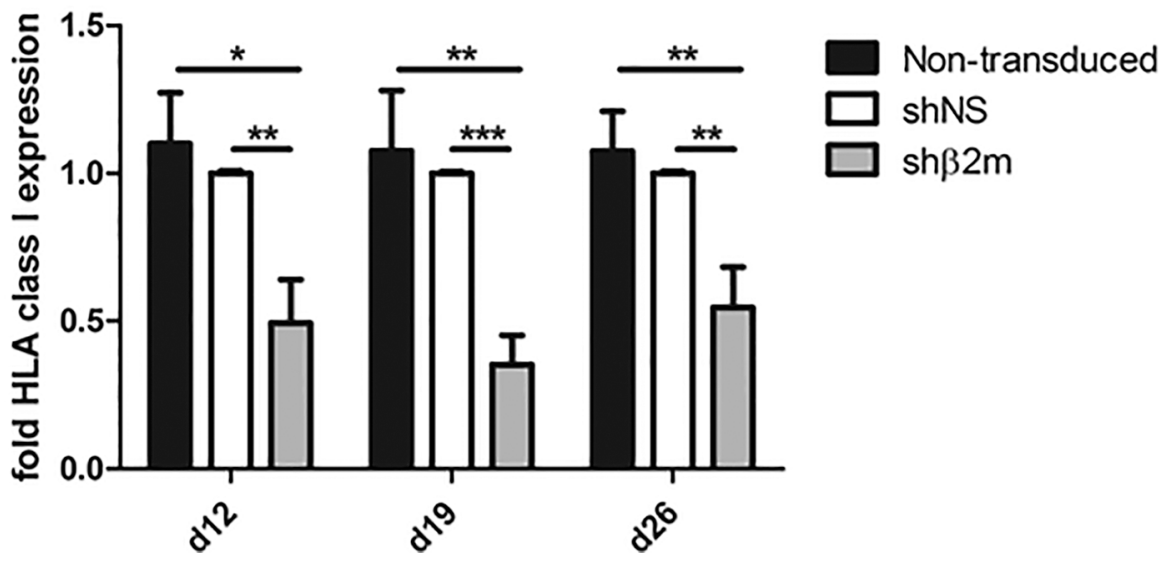

Figure 4. Megakaryocytes (MKs) differentiated from HLA-universal iPSCs remain silenced for HLA class I. (A) Relative levels of $\beta 2 \mathrm{~m}$ transcripts in shNS- or sh $\beta 2 \mathrm{~m}$ - expressing and nontransduced MKs assessed by real time PCR. (B) Surface expression of HLA class I molecules in nontransduced, nonsilenced and silenced MKs analyzed by flow cytometry. Cells were harvested and stained with HLA-specific antibody w6/32. Both graphs depict means and SD of three independent experiments. ${ }^{* * *} p \leq 0.001,{ }^{* *} p \leq 0.01,{ }^{*} p \leq 0.05$.

\section{HLA-Universal MKs Generate PLTs in a Refractoriness Mouse Model}

Transfusion of MKs has been proposed as an alternative to PLT transfusion. However, in prior studies, the numbers of BM MKs collected were insufficient for a therapeutically effective transfusion $(22,23)$. Transfusion of NOD/SCID/IL$2 \mathrm{R}^{-/-}$mice with either HLA-expressing or HLA-silenced iPSC-derived MKs resulted in the production of PLTs, which were detectable in the murine circulation. However, in presence of an anti-HLA-A*02 antibody, the frequencies of fully HLA-expressing PLTs
$(0.15 \% \pm 0.03 \%)$ detectable $1 \mathrm{~h}$ after transfusion were already lower than in the absence of the antibody $(0.24 \% \pm 0.03 \%)$. Notably, $4 \mathrm{~h}$ after transfusion, a significant reduction in shNS-PLT frequencies $(0.07 \% \pm 0.02 \%$, $p<0.01)$ in comparison to those detected in mice without anti-HLA-A ${ }^{*} 02$ treatment $(0.20 \% \pm 0.05 \%)$ were observed. In contrast, no significant differences in PLTs derived from HLA-silenced iPSCs were detected $1 \mathrm{~h}$ after transfusion in anti-HLA- ${ }^{*} 02$ - treated mice $(0.25 \% \pm$ $0.07 \%$ ) in comparison to nontreated mice $(0.20 \% \pm 0.06 \%)$. Also, $4 \mathrm{~h}$ after transfusion of HLA-silenced PLTs, no significant differences of HLA-silenced PLT frequencies among nontreated $(0.16 \% \pm 0.08 \%)$ and anti-HLA-A ${ }^{*} 02-$ antibody-treated mice $(0.12 \% \pm 0.09 \%)$ were observed (Figure 8). In biodistribution assays, we have analyzed different mice organs (heart, lung, spleen, BM) for the presence of human MKs after 1 or $14 \mathrm{~d}$ post injection. No human MKs were detectable in the organs indicating that the transfused MKs remained and produced PLTs only in the mouse blood circulation (data not shown). These data indicate the capacity of HLA-universal MKs to produce PLTs in vivo.

\section{DISCUSSION}

In this study, we describe the generation of a HLA-universal iPSC line, which may serve as an alternative cell source for the differentiation of low immunogenic cell products. Our previous studies demonstrated that silencing the expression of HLA prevents allogeneic immune responses in vitro and in vivo even in the absence of immunosuppression. Due to technical and economical constraints, the use of iPSC-derived products will likely occur in an allogeneic setting. Nevertheless, the application of such products is associated with the risk of immune rejection due to nonpermissive HLA and mHA mismatches. Currently, attempts in establishing large iPSC haplobanks are running. Such iPSC haplobanks may cover the most frequent HLA haplotypes, but will not be able to fully match mHA between iPSC-derived products and recipients $(3,13,14)$. Hence, the generation of HLA-universal iPSC lines may overcome the need for large iPSC haplobanks as they can be used for the generation of HLA-silenced cell products which are not recognized by the recipient's immune system. In this report, we have shown the feasibility to generate permanently HLA class I-silenced iPSCs using RNAi. In our previous studies, we have demonstrated in different models and for different cell types that silencing HLA class I expression by more than $90 \%$ trigger NK cell cytotoxicity $(15,16)$. 
A

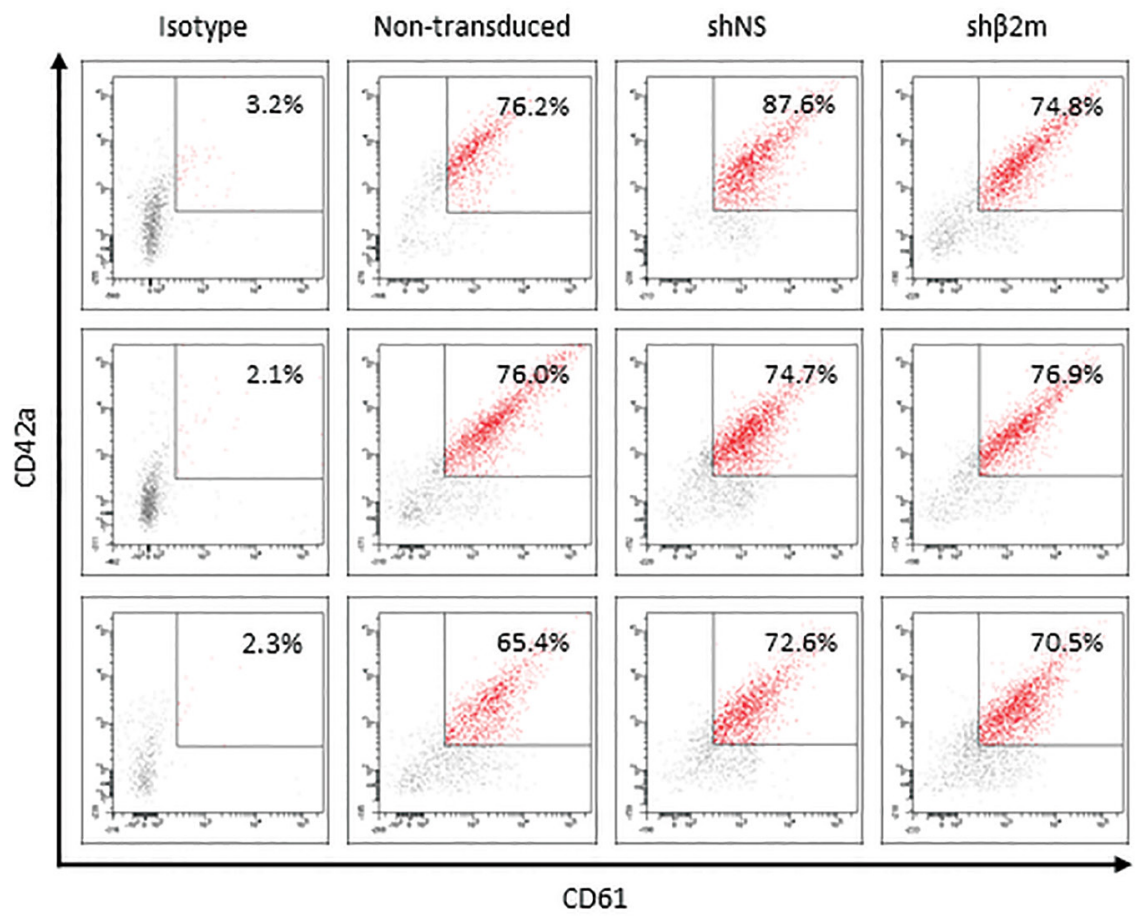

B

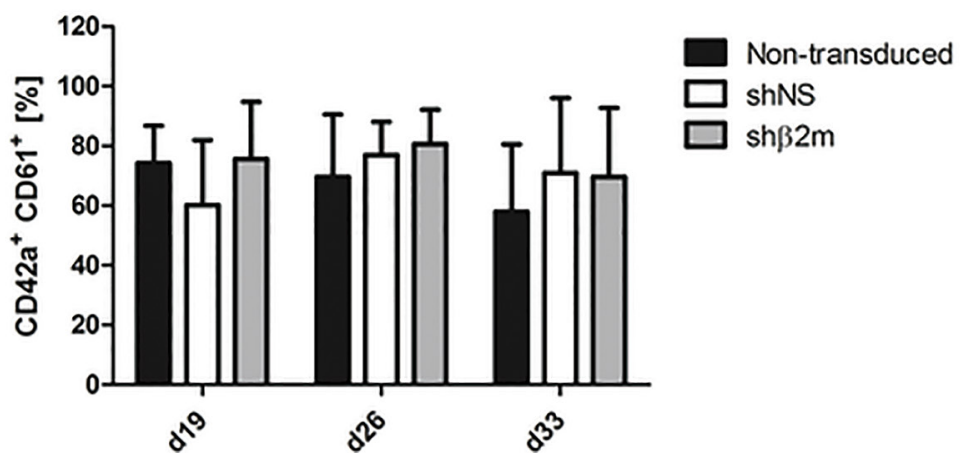

Figure 5. Characterization of HLA class I-silenced iPSC-derived platelets (PLTs). Nontransduced iPSCs and iPSCs expressing either a control nonspecific short-hairpin RNA (shNS) or a shRNA targeting $\beta 2$-microglobulin transcripts (sh $\beta 2 \mathrm{~m}$ ) were cultured for $33 \mathrm{~d}$ and analyzed weekly from d 12 for PLT differentiation (four time points). (A) PLTs were identified by selection of a CD41 ${ }^{+}$(GPIlb) population. Shown is the percentage of CD42a (GPIX) $\mathrm{CD} \mathrm{1}^{+}$(GPIIla) co-expression of $\mathrm{CD} 41^{+}$cells. Representative flow cytometry dot plots are shown for each condition and time point. (B) Mean and SD of CD42 $\mathrm{a}^{+} \mathrm{CD} 61^{+}$ cells of four independent experiments.

These observations suggest that powerful gene editing technologies such as zinc finger nucleases (ZFN), transcription activator-like effector nucleases (TALENs) or Clustered Regularly Interspaced Short
Palindromic Repeats (CRISPR), which cause a complete gene knockout, may not be suitable to reduce cell immunogenicity as a complete lack of HLA class I expression is expected to trigger NK cell cytotoxicity. In this study, RNAi-mediated HLA class I silencing allowed the generation of a HLA-universal iPSC line with the capacity to serve as a cell source for low immunogenic cell products.

PLT transfusion is a widespread therapeutic strategy to treat life-threatening conditions caused by severe and persistent thrombocytopenia due to prolonged BM aplasia or insufficient PLT function secondary to genetic disorders or malignancy (24). Despite the key role of PLTs in the maintenance of hemostasis, they were shown to regulate multiple processes such as angiogenesis, cell differentiation, cell migration, immune responses and tissue remodeling (25-27). More recently, those PLT unique features have been exploited in the development of novel therapies such as in supporting wound healing, tissue regeneration or as drug carriers (28-31). Due to the population aging, a significant increase on PLT demand for all those therapeutic approaches is expected. It will be difficult to fulfil this necessity only with human volunteer donors (32), therefore, it is desirable to establish alternative PLT sources. To solve the problem of PLT availability, we and others have established different protocols to generate functional PLTs in vitro $(9,19,33-35)$. Nevertheless, PLT transfusion is associated with risks and adverse effects. PLT transfusion refractoriness is a serious clinical condition because it leads to hemorrhagic events that might be fatal for the patient. The most important immune cause for refractoriness is alloimmunization against PLT antigens. About $7 \%$ to $55 \%$ of the patients receiving PLT transfusions develop antibodies specific for HLA class I epitopes. In contrast, only $2 \%$ of the transfused patients develop antibodies against other human PLT antigens $(3,36,37)$. Several strategies including intravenous administration of immunoglobulin (IVIG) or plasmapheresis have been used for the management of PLT transfusion refractoriness. However, those strategies have rendered controversial results. Another attempt to overcome refractoriness to 


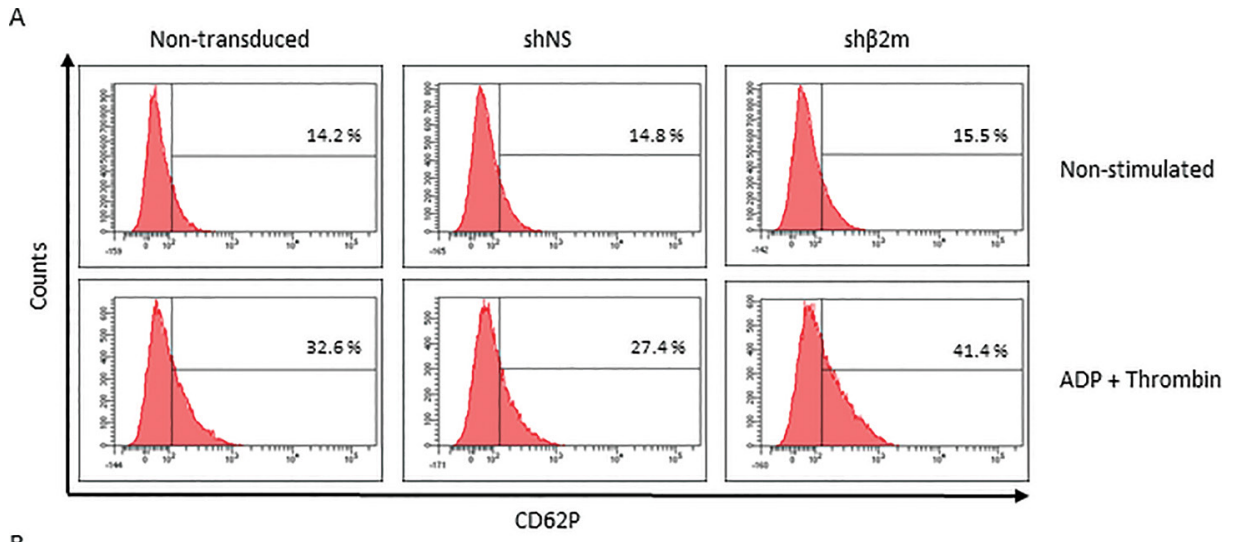

B

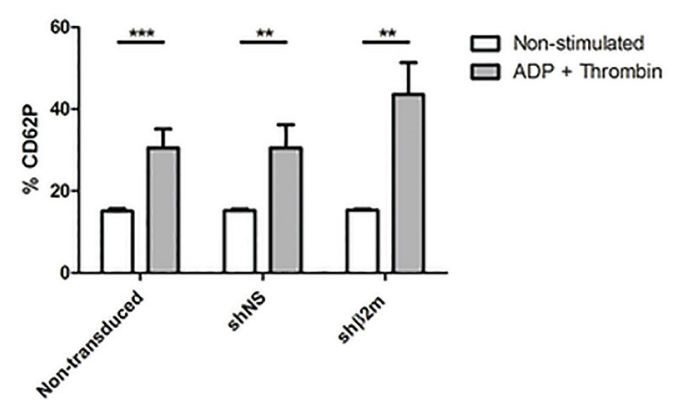

Figure 6. Functional characterization of HLA-universal platelets (PLTs). PLTs derived from nontransduced iPSCs or iPSCs expressing a nonspecific shRNA (shNS) or a $\beta 2$ microglobulin-specific shRNA (sh $\beta 2 \mathrm{~m}$ ) were stimulated with ADP and thrombin. The upregulation of CD62P (P-selectin) was analyzed. $(A)$ Representative histogram of CD62P expression. (B) Graph displays mean and SD of the frequencies of CD62P as detected in three independent experiments. ${ }^{* * *} p \leq 0.001,{ }^{* *} p \leq 0.01$.

A

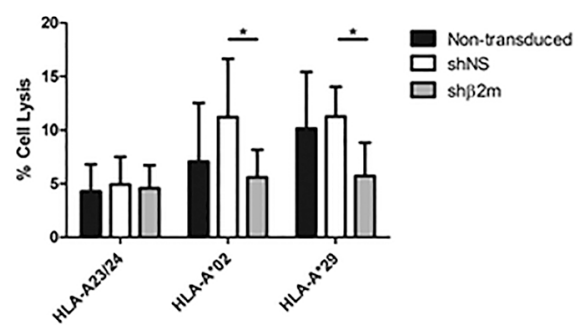

B

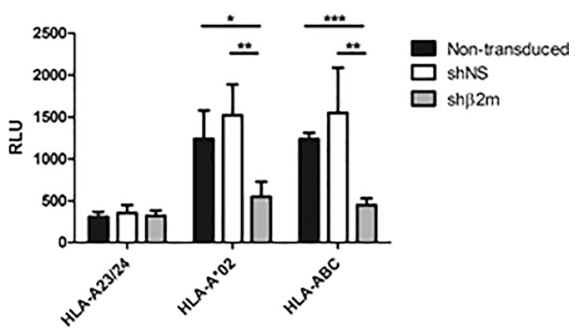

PLT transfusion is based on the selection of HLA-matched PLTs. Nevertheless, this approach is limited by the availability and is costly and time consuming. In addition, recent studies have shown that $20 \%-50 \%$ of severe alloimmunized patients remain refractory even after transfusion of HLA-matched PLTs (21). Previously, we have reported the generation of HLA-universal PLTs derived from $\mathrm{CD}_{3} 4^{+}$progenitor cells, which demonstrated the capacity to escape refractoriness conditions in vivo (10). Here, we have established a protocol for the generation of PLTs from a HLA-universal and virtually unlimited cell source (iPSCs) under xeno-free and defined conditions to facilitate their future translation into clinical application. In addition, first attempts to use a good manufacturing practice conform vector ( $p$ RRL.PPT.EFS.dLNGFR. pre) for silencing HLA expression were performed (Supplementary Figures S4 and S5). The efficient differentiation of HLA-universal MKs and PLTs was achieved. Silencing HLA class I expression did not affect the differentiation capacity of the iPSC line into MKs and PLTs. Notably, a significant reduction of HLA class I antigens was still detectable in MKs and PLTs. Similarly to our previous results obtained using HLA-universal MKs and PLTs derived from $\mathrm{CD} 34^{+}$progenitor cells (10), also HLA-universal iPSC-derived MKs were protected from antibody-mediated CDC and ADCC, which indicate their capacity to survive under PLT transfusion refractoriness conditions. Our results are further supported by the recent study of Meinke et al., reporting that the reduction of HLA complexes from the PLT surface with acid treatment confers protection against CDC and monocyte-mediated phagocytosis (38). Hence, this study demonstrates the feasibility to generate low immunogenic MKs and PLTs from iPSCs. Importantly, HLA-universal PLTs proved to be functional in stimulation assays using ADP and thrombin as agonists. After exposure to ADP and thrombin, HLA-universal PLTs were able 
A

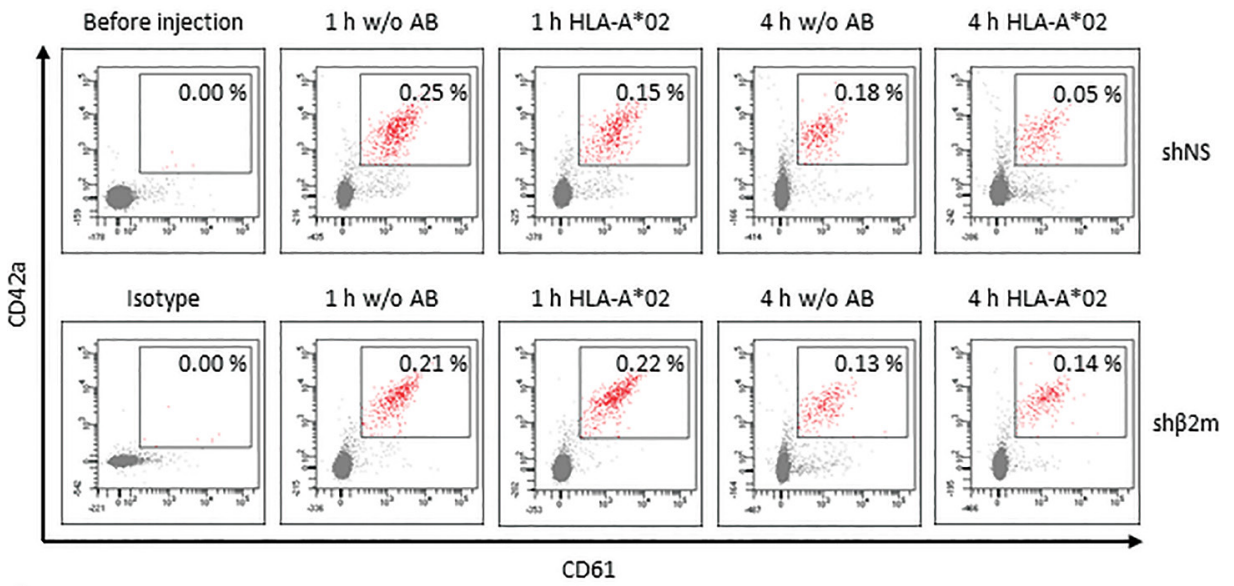

B

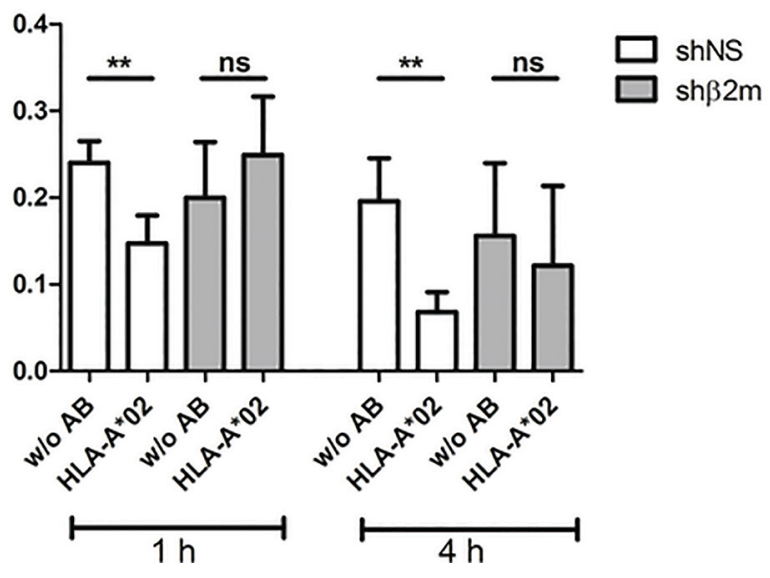

Figure 8. HLA-universal megakaryocytes (MKs) produce platelets (PLTs) after transfusion into a mouse model. Mice were transfused with $3 \times 10^{6} \mathrm{HLA}$-universal iPSC-derived MKs by tail vein injection. Twenty minutes before transfusion of MKs, the mice get immunized by injection of anti-HLA-A*02 antibody. Nonimmunized mice served as a control. Frequencies of human PLTs were detected before transfusion as well as 1 and $4 \mathrm{~h}$ after MK infusion by flow cytometric analysis of the PB. (A) Representative dot plots displaying human PLTs detected in the mouse circulation. The gate indicates the frequency of human PLTs. (B) The graph shows mean and SD of the PLT frequencies detected after HLA-universal MK transfusion in three independent experiments. ${ }^{* *} p \leq 0.01$.

to upregulate CD62P and to aggregate. These results are in concordance with previous studies, which have shown that iPSC-derived HLA-expressing PLTs are functional $(9,39)$. However, in contrast to HLA-expressing PLTs, HLA-universal PLTs have the capacity to survive in presence of alloantibodies. Feng et al. have used TALEN technology to knockout $\beta 2 \mathrm{M}$, thus generating completely
HLA-deficient iPSCs. However, the same group has later recognized that $\beta 2 \mathrm{M}^{-/-}$ cells are susceptible targets for NK cell activity and discuss the co-engineering of iPSCs with the HLA-E molecule, which is known to inhibit NK cell activity (40). Based on our studies, we believe that RNAi might be a superior tool to reduce the immunogenicity of iPSC-derived cell products because silencing HLA expression confers protection against HLA-specific alloimmune responses, but the residual HLA class I expression alone is sufficient to prevent NK cell cytotoxic activity without further requirement for iPSC genetic engineering $(15,16)$.

As an alternative to PLT transfusion to treat thrombocytopenia, the transfusion of their precursors (MKs) has been proposed (22). Poncz et al. have previously demonstrated the feasibility to increase PLT counts in the blood circulation upon MK transfusion. PLTs derived from MK transfusion have shown a typical size and phenotype and were capable to incorporate in thombi. However, the technical difficulties to collect and expand BM MKs ex vivo have limited the feasibility to apply this strategy in the clinics (41). Nevertheless, those hurdles are now overcome by the possibility to generate many HLA-silenced MKs in vitro. In a mouse model, we have demonstrated that transfused HLA class I-silenced iPSC-derived MKs are able to produce PLTs in vivo. PLTs were already detectable $1 \mathrm{~h}$ after MK transfusion. MK transfusion showed to be well tolerated by the mice and no adverse effect was observed. Previously, it has been suggested that transfused MKs may produce PLTs from the pulmonary vasculature (41). In this study, infused iPSC-derived MKs were not found in the lung. In further biodistribution assays, the MKs were also not detected in organs such as heart, spleen or BM (data not shown). These results suggest that the transfused MKs may produce PLTs in the peripheral circulation, however further studies will be required to comprehensively investigate this issue. Altogether, these results indicate that $\mathrm{MK}$ transfusion may constitute an alternative strategy to PLT transfusion.

\section{CONCLUSION}

In summary, the use of HLA-universal iPSCs may constitute a reliable source for the generation of donor-independent low immunogenic cell products for regenerative purposes. Our study shows the efficient generation of HLA-universal 
MKs and PLTs with the capacity to survive under refractoriness conditions. HLA-universal MKs showed the capability to form PLTs in vitro and in vivo and have the potential to become an important component in the management of non, weak or severe alloimmunized thrombocytopenic patients.

\section{ACKNOWLEDGMENTS}

We are grateful to Sarah Beyer and Elena Reinhard for the excellent technical assistance. This work was supported by funding from the German Foundation for Transfusion Medicine and the German Research Foundation (DFG) for the Cluster of Excellence REBIRTH (From Regenerative Biology to Reconstructive Therapy) (EXC 62).

\section{DISCLOSURE}

The authors declare they have no competing interests as defined by Molecular Medicine, or other interests that might be perceived to influence the results and discussion reported in this paper.

\section{REFERENCES}

1. Fasano RM, et al. (2014) Persistence of recipient human leucocyte antigen (HLA) antibodies and production of donor HLA antibodies following reduced intensity allogeneic haematopoietic stem cell transplantation. Br. J. Haematol. 166:425-34. DOI $10.1111 /$ bjh. 12890

2. Stanworth SJ, Navarrete C, Estcourt L, Marsh J. (2015) Platelet refractoriness - practical approaches and ongoing dilemmas in patient management. Br. J. Haematol. 171:297-305. DOI 10.1111/bjh.13597

3. Rebulla P. (2005) A mini-review on platelet refractoriness. Haematologica. 90:247-53.

4. Pena JR. (2014) Anti-HLA alloantibodies in surgical patients refractory to platelet transfusion. Am. J. Hematol. 89:E133-E137. DOI 10.1002/ajh.23757

5. Beligaswatte A. (2013) The mean fluorescence intensities of anti-HLA antibodies detected using micro-bead flow cytometry predict the risk of platelet transfusion refractoriness. Br. J. Haematol. 162:409-12. DOI 10.1111/bjh.12369

6. Pavenski K, Freedman J, Semple JW. (2012) HLA alloimmunization against platelet transfusions: Pathophysiology, significance, prevention and management. Tissue Antigens. 79:237-45. DOI 10.1111/j.1399-0039.2012.01852.x

7. Conti FM, et al. (2014) Induction of platelet refractoriness after myeloablative unrelated allogeneic hematopoietic peripheral blood progenitor cell transplant from HLA-sensitized female donor. Transfusion. 54:3015-17. DOI $10.1111 /$ trf. 12856

8. DHHS. (2011) The 2011 national blood collection and utilization survey report. National Blood Collection and Utilization Report. Washington (DC): DHHS.

9. Feng Q, et al. (2014) Scalable generation of universal platelets from human induced pluripotent stem cells. Stem Cell Reports. 3:817-831. DOI 10.1016/j.stemcr.2014.09.010

10. Gras C, et al. (2013) HLA-universal platelet transfusions prevent platelet refractoriness in a mouse model. Hum. Gene Ther. 24:1018-28. DOI 10.1089/hum.2013.074

11. Ackermann M, Liebhaber S, Klusmann JH, Lachmann N. (2015) Lost in translation: Pluripotent stem cell-derived hematopoiesis. EMBO Mol. Med 7:1388-1402. DOI 10.15252/ emmm.201505301

12. Kiskinis E, Eggan K. (2010) Progress toward the clinical application of patient-specific pluripotent stem cells. J. Clin. Invest. 120:51-59. DOI 10.1172/ JCI40553

13. Gourraud PA, Gilson L, Girard M, Peschanski M. (2012) The role of human leukocyte antigen matching in the development of multiethnic "haplobank" of induced pluripotent stem cell lines. Stem Cells. 30:180-6. DOI 10.1002/stem.772

14. de Rham C, Villard J. (2014) Potential and limitation of HLA-based banking of human pluripotent stem cells for cell therapy. J. Immunol. Res. 2014:518135. DOI 10.1155/2014/518135

15. Figueiredo C, et al. (2013) MHC universal cells survive in an allogeneic environment after incompatible transplantation. Biomed Res. Int. 2013:796046. DOI 10.1155/2013/796046

16. Wiegmann B, et al. (2014) Prevention of rejection of allogeneic endothelial cells in a biohybrid lung by silencing HLA-class I expression. Biomaterials. 35:8123-33. DOI 10.1016/j.biomaterials.2014.06.007

17. Haase A, et al. (2009) Generation of induced pluripotent stem cells from human cord blood. Cell Stem Cell. 5:434-41. DOI 10.1016/j. stem.2009.08.021

18. Figueiredo C, Seltsam A, Blasczyk R. (2006) Class-, gene-, and group-specific HLA silencing by lentiviral shRNA delivery. J. Mol. Med. 84:425-37. DOI 10.1007/s00109-005-0024-2

19. Figueiredo C, et al. (2010) Generation of HLA-deficient platelets from hematopoietic progenitor cells. Transfusion. 50:1690-1701. DOI 10.1111/j.1537-2995.2010.02644.x

20. Rubak P, Nissen PH, Kristensen SD, Hvas AM. (2015) Investigation of platelet function and platelet disorders using flow cytometry. Platelets. 27:66-74. DOI 10.3109/09537104.2015.1032919

21. Cid J, et al. (2015) Rituximab, plasma exchange and intravenous immunoglobulins as a new treatment strategy for severe HLA alloimmune platelet refractoriness. Platelets. 26:190-4. DOI 10.3109/09537104.2014.895922
22. Decaudin D, et al. (2004) Ex vivo expansion of megakaryocyte precursor cells in autologous stem cell transplantation for relapsed malignant lymphoma. Bone Marrow Transpl. 34:1089-1093. DOI 10.1038/sj.bmt.1704675

23. Gehling UM, et al. (1997) Ex vivo expansion of megakaryocyte progenitors: Effect of various growth factor combinations on CD34+ progenitor cells from bone marrow and G-CSF-mobilized peripheral blood. Exp. Hematol. 25:1125-39.

24. Mishima $Y$, et al. (2015) Effects of universal vs bedside leukoreductions on the alloimmunization to platelets and the platelet transfusion refractoriness. Transfus. Apher. Sci. 52:112-21. DOI 10.1016/j.transci.2014.11.001

25. Anitua E, Andia I, Ardanza B, Nurden P, Nurden AT. (2004) Autologous platelets as a source of proteins for healing and tissue regeneration. Thromb. Haemost. 91:4-15. DOI 10.1267/ THRO04010004

26. Semple JW, Freedman J. (2010) Platelets and innate immunity. Cell. Mol. Life Sci. 67:499-511. DOI 10.1007/s00018-009-0205-1

27. Cervelli V, et al. (2012) Platelet-rich plasma greatly potentiates insulin-induced adipogenic differentiation of human adipose-derived stem cells through a serine/threonine kinase Aktdependent mechanism and promotes clinical fat graft maintenance. Stem Cells Transl. Med. 1:206-20. DOI 10.5966/sctm.2011-0052

28. Femminella B, et al. (2015) Clinical comparison of platelet-rich fibrin and a gelatin sponge in the management of palatal wounds following epithelialized free gingival graft harvest: A randomized clinical trial. J. Periodontol. 87:1-17. DOI 10.1902/jop.2015.150198

29. Fernandez-Moure JS, et al. (2015) Platelet rich plasma enhances tissue incorporation of biologic mesh. J. Surg. Res. 199:412-19. DOI 10.1016/j. jss.2015.06.034

30. Iesari S, et al. (2015) Infected nonhealing wound in a kidney transplant recipient: Successful treatment with topical homologous platelet-rich gel. Exp. Clin. Transplant. DOI 10.6002/ ect.2014.0236 [Epub ahead of print 2015 Jun 15]

31. Sarkar S, Alam MA, Shaw J, Dasgupta AK. (2013) Drug delivery using platelet cancer cell interaction. Pharm. Res. 30:2785-94. DOI 10.1007/ s11095-013-1097-1

32. Estcourt LJ. (2014) Why has demand for platelet components increased? A review. Transfus. Med. 24:260-268. DOI 10.1111/tme.12155

33. Nakamura S, et al. (2014) Expandable megakaryocyte cell lines enable clinically applicable generation of platelets from human induced pluripotent stem cells. Cell Stem Cell. 14:535-48. DOI 10.1016/j.stem.2014.01.011

34. Takayama N, et al. (2008) Generation of functional platelets from human embryonic stem cells in vitro via ES-sacs, VEGF-promoted structures that concentrate hematopoietic progenitors. Blood. 111:5298-306. DOI 10.1182/blood2007-10-117622 
35. Norol F, et al. (1998) Effects of cytokines on platelet production from blood and marrow CD34+ cells. Blood. 91:830-43.

36. van Marwijk Kooy M, van Prooijen HC, Moes M, Bosma-Stants I, Akkerman JW. (1991) Use of leukocyte-depleted platelet concentrates for the prevention of refractoriness and primary HLA alloimmunization: A prospective, randomized trial. Blood. 77:201-5.

37. Kiefel V, Konig C, Kroll H, Santoso S. (2001) Platelet alloantibodies in transfused patients. Transfusion. 41:766-70.

38. Meinke S, et al. (2015) Platelets made HLA deficient by acid treatment aggregate normally and escape destruction by complement and phagocytes in the presence of HLA antibodies. Transfusion. 56:370-82. DOI 10.1111/trf.13350

39. Takayama N, et al. (2010) Transient activation of c-MYC expression is critical for efficient platelet generation from human induced pluripotent stem cells. J. Exp. Med. 207:2817-30. DOI 10.1084/ jem.20100844

40. Thon JN, Medvetz DA, Karlsson SM, Italiano JE, Jr. (2015) Road blocks in making platelets for transfusion. J. Thromb. Haemost. 13 Suppl 1: S55-62. DOI 10.1111/jth.12942

41. Fuentes R, et al. (2010) Infusion of mature megakaryocytes into mice yields functional platelets. J. Clin. Invest. 120:3917-22. DOI 10.1172/JCI43326

Cite this article as: Börger A-K, et al. (2016) Generation of HLA-universal iPSC-derived megakaryocytes and platelets for survival under refractoriness conditions. Mol. Med. 22:274-85. 